\title{
Orthodontic Treatment with Clear Aligners and The Scientific Reality Behind Their Marketing: A Literature Review
}

\author{
İpek Tamer (D), Evren Öztaş (D), Gülnaz Marşan (D)
}

Department of Orthodontics, Istanbul University Istanbul School of Dentistry, Istanbul, Turkey

Cite this article as: Tamer I, Öztaş E, Marşan G. Orthodontic Treatment with Clear Aligners and The Scientific Reality Behind Their Marketing: A Literature Review. Turk J Orthod 2019; 32(4): 241-6.

\section{ABSTRACT}

As the demand for esthetic treatments is increasing, more people are seeking alternatives to fixed orthodontic appliances. Clear aligners are an esthetic and comfortable option for orthodontic treatment and have gained immense popularity over the last decade. This review will highlight the increasing popularity of clear aligners by describing some aligner systems frequently used today. The scope, limitations, effectiveness, efficacy, and stability of treatment results achieved with this method will be discussed. Further, this paper will assess the possible side effects caused by clear aligner treatment.

Keywords: Orthodontics, orthodontic treatment, aesthetics, clear aligners

\section{INTRODUCTION}

The increase in the number of adult orthodontic patients has prompted an upsurge in the demand for esthetic and comfortable alternatives to conventional fixed appliances (1-4). Clear aligners that satisfy this demand are also prone to rapid technological improvements in aligner materials and production techniques (1). Developments in clear aligner technologies have increased the number and complexity of cases treated with this method (5). Clear aligners provide an esthetic and comfortable treatment experience, facilitate oral hygiene, cause less pain as compared to fixed orthodontic appliances, reduce the number and duration of appointments, and require less emergency visits (6-8). However, the expense in production, dependency on patient cooperation, and the inability to treat certain malocclusions limit the usage of clear aligners (1, 3, 5, 9-12). An electronic search in the English language was conducted in December 2017 in the following electronic databases: Google Scholar, Web of Science, and PubMed. We checked the bibliographies of included papers and relevant review articles. Only prospective and retrospective human studies were included while animal studies, editorials, and case reports were excluded.

\section{Clinical and Research Consequences}

According to production methods, clear aligner systems can be broadly grouped into two categories; aligners made from thermoplastic materials via manual set up and systems using CAD-CAM technologies to design and produce aligners (13). It is impossible to describe each system in this review; therefore, the most widely used systems will be discussed.

\section{Aligners Produced by Manual Set up}

The manual approach is a labor-intensive process, requiring manual repositioning of the teeth, wax setting, and production of vacuum-formed retainers. This approach allows the fabrication of aligners easily in laboratory conditions in a cost-effective manner. It also facilitates the follow-up process of the treatment and allows the orthodontist to make the necessary treatment changes at an earlier stage. 
Full arch impressions are taken using polyvinyl siloxane material and a working cast is obtained. On the working casts, teeth that are planned to be moved in each aligner are determined and removed from the cast using a $0.25-\mathrm{mm}$ handsaw. The separated target teeth are then moved to the desired position and are fixed using the block-out wax. If needed, the interproximal reduction is performed at this stage. After this realignment, plastic sheets are molded on the setup model using a pressure molding machine or vacuum machine. Following the final trimming procedures, a 3-piece set of aligners are delivered to the patient (19).

Aligners are produced in various thickness levels (0.020-inch, 0.025 -inch, or 0.030 -inch). The use of gradually thickening aligners provides more control on tooth movement and reduces the pain caused by orthodontic forces. With one set of impressions, two or three aligners of various thickness levels are produced, and the patient is instructed to use each aligner for 10 to 15 days. The aligners are fabricated from a new working cast and obtained from a new impression taken at each visit, which allows the clinician to modify the treatment plan throughout the course of treatment, and to be able to follow the progression of tooth movement $(14,15)$. Clear Aligner system CA (Scheu Dental, Germany) is an example of aligner systems requiring a manual setup. In this system, a computer program, Aligner Aid (AAP, IV- Tech, South Korea) is used to accurately measure the tooth movement obtained. It is possible to measure the tooth movement by naked eye visualization, but this program is recommended when more than one tooth is to be moved. Before the initial setup is made, a photo of the working cast is taken using a digital camera, and this photo is superimposed over the photo of the setup model. The program measures the distance and angle of the teeth that are to be moved and recommends that the total teeth movement obtained in one set of aligners be limited to $5 \mathrm{~mm}(14,15)$.

\section{Aligners Produced by CAD-CAM Technologies}

The incorporation of digital technology has revolutionized the practice and appliances used in orthodontics. As in other fields of dentistry, CAD-CAM systems have become involved in orthodontics and aligner treatment.

Invisalign ${ }^{\circ}$ being the best-known aligner system has become a generic name for other high-quality systems using CAD-CAM technology This system is known to be the most sophisticated and most commonly used clear aligner technology currently available (1). In 1999, the Invisalign ${ }^{\circ}$ system was introduced to the orthodontic market to treat mild malocclusions only; however, the development of different attachments and auxiliaries now enables Invisalign ${ }^{\circ}$ system to perform major tooth movements and treat more complex cases such as those requiring premolar extraction (16-18). Aligners in Invisalign" system are designed and produced using CAD-CAM technology (13). The combination of computerized virtual treatment planning, and stereolithographic prototyping technology for manufacturing gives Invisalign ${ }^{\circ}$ a leading role in aligner therapy $(4,5,19,20)$.Today, Align Technology continues to be a leader in the market, and Invisalign has become a household name for aligners produced by computers since more than 4 million people are treated by this system. Meanwhile, literature research in 2015 revealed approximately 27 different clear aligner systems on offer, a number that continues to increase rapidly (21-26).Companies like Orthero, ECligner, EON Aligner, and Clear Correct are examples of other aligner systems created using computer technology

\section{Biomechanics of Aligner Treatment}

Understanding the mechanics of tooth movement using aligners could lead to the more appropriate selection of patients and more accurate treatment sequencing, leading to better results (10).

Tooth movement mechanism with clear aligners can be explained from two different perspectives: the displacement driven system and the force driven system $(10,21)$. The displacement driven system mainly controls simple movements such as tipping or minor rotations. Aligners are formed according to the position of the tooth in the next staged location and the tooth continues to move until it lines up with the aligner. This system is known to be less effective in controlling tooth movement and is insufficient in producing root movements. The force driven system, however, requires biomechanical principles to facilitate tooth movement. Aligners are designed to apply desired forces on the tooth. The shape of aligners to produce such forces is not necessarily the same as the shape of the tooth. The movement required for each individual tooth, mechanical principles to accomplish this movement, and the aligner shape are determined via Clincheck (Align Technology, Santa Clara, CA, USA) software. The aligner shape is altered via pressure points or power ridges in order to apply the desired forces $(4,10,21)$. Pressure points lead to more difficult uprighting and intrusion movements, whereas power ridges control axial root movements and torque (1, 27) (Figure 1, 2).

Despite the alterations in the shape of the aligner, movements such as root paralleling, extrusion, and rotation were still difficult to obtain using aligners until Align Tech. (Align Technology, Santa Clara, CA, USA) introduced smart force attachments for the Invisalign ${ }^{\circ}$ system. These attachments are small composite bulges designed to produce a force system favorable for the designed movement. Their position and shape are determined via Clincheck software according to the movement to be obtained. Extrusion attachment, rotation attachment, and root control attachments are currently used. Extrusion of a single tooth is moderately difficult using clear aligners when compared to fixed-appliance systems, however, some auxiliaries such as buttons and elastics can be used to facilitate this movement. Also, the extrusion of a group of teeth (i.e., maxillary incisors) can be performed using aligners (Figure 3).

The use of temporary anchorage devices in combination with clear aligners further widened the range of treatments possible with aligners $(27,28)$.

\section{Scope and Limitations of Treatment with Clear Aligners}

Although the number and complexity of cases treated with clear aligners continue to increase, it is impossible to treat all kinds of malocclusions with this system. Clear aligners are convenient in mild to moderate crowding or diastema, posterior expansion, 
intrusion of one or two teeth, lower incisor extraction cases, and distal tipping of molars. Movements like extrusion, correction of severe rotations, molar uprighting, and closure of extraction spaces are known to be more challenging to accomplish with aligners. Even so, incisor extrusion, molar transition, and closure of extraction spaces are possible with the use of attachments in the Invisalign system $(4,9,18,23)$.

\section{Efficacy and Efficiency of Clear Aligners}

As the demand and interest toward the clear aligner system continue to grow, questions regarding the efficacy of the system remain $(20,29)$. To date, published data include little clinical research on the effectiveness and efficacy of clear aligners $(1,30)$. Previous literature primarily includes case reports or descriptions of the product, making it difficult to objectively characterize the efficacy of clear aligner systems $(29,30)$.

\section{Clinical Effectiveness of Clear Aligners}

In 2005, Djeu et al. (31) conducted the first retrospective cohort study on the effectiveness of clear aligners, which compared the treatment results of Invisalign patients with the results of conventional fixed braces using the American Board of Orthodontics grading system. They reported that both systems are equally effective in space closure, marginal ridge alignment and, root paralleling; however, the Invisalign ${ }^{\circ}$ system is deficient in the correction of anteroposterior discrepancies, providing occlusal contacts, and posterior torque.
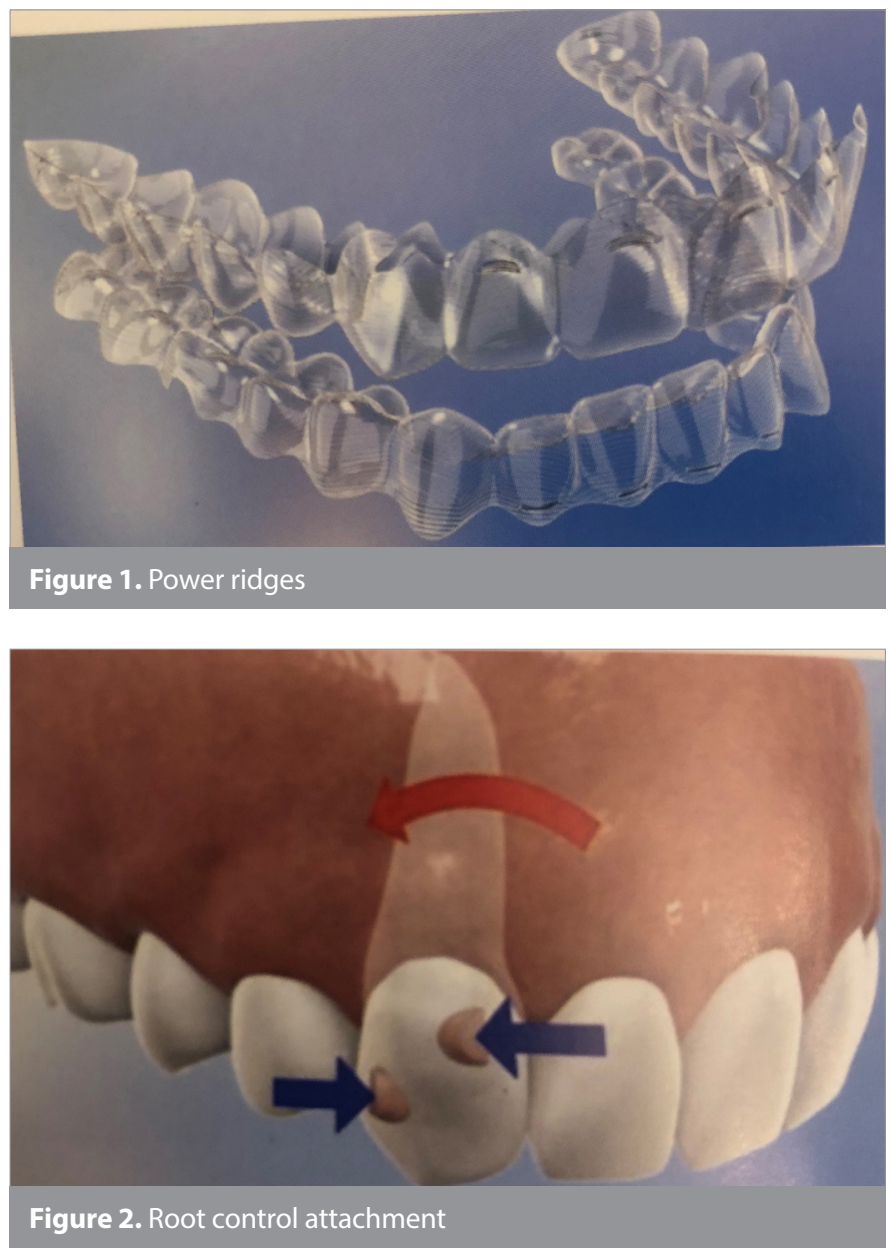

Parallel to the previous study, Kassas et al. (32) reported that the clear aligner system is effective in leveling and aligning arches in mild and moderate cases and in correcting buccolingual inclinations effectively, however, it is not sufficient for providing ideal occlusal contacts. The deterioration in occlusal contacts is caused by the thickness of aligners, which interferes with the settling of the occlusal plane. Kravitz et al. (20) evaluated the accuracy of tooth movement obtained by the Invisalign "system and reported that only $41 \%$ of the predicted tooth movement was achieved. The most effective movement was lingual constriction (47.1\%), the least accurate was extrusion (29.6\%), and only $33 \%$ of predicted rotation correction was achieved.

The lower canine is the most difficult tooth to control. Weihong et al. (33) evaluated the effectiveness of the Invisalign system on mild to moderate cases treated with premolar extractions and compared the treatment results obtained with fixed appliances. Their results revealed that both systems can be used in the treatment of extraction cases, and that root angulation attained with clear aligners are adequate when proper attachments are to be used. However, it should be kept in mind that treating extraction cases requires experience and extensive knowledge of the system (34, 35). The majority of the literature focuses on the effects obtained via the Invisalign system. Yıldırım et al. (36) investigated the efficacy of tooth movements obtained with clear aligner appliances. In their study, retrusion was found to be the most accurately obtained tooth movement followed by a rotation, fan-type expansion, and protrusion respectively. Retrusion of mandibular central incisors is considered to be the most accurate single-tooth movement, whereas the rotation of mandibular canine is the least accurate movement. Due to the lack of scientific data and poor methodologies of the available studies, results should be interpreted with caution. Further research is required in this field $(19,35)$.

\section{Time Efficiency of Clear Aligners}

Time efficiency is an important outcome to consider for private practice orthodontists because spending less time with one patient in the clinic and completing the treatment earlier both pleases the current patient and allows the orthodontist to treat more patients (37). Bushang et al. (38) investigated the difference between conventional fixed appliances and Invisalign aligners in terms of total treatment time and chair time in non-extraction patients. Total treatment time was found to be $67 \%$ lesser in the In-

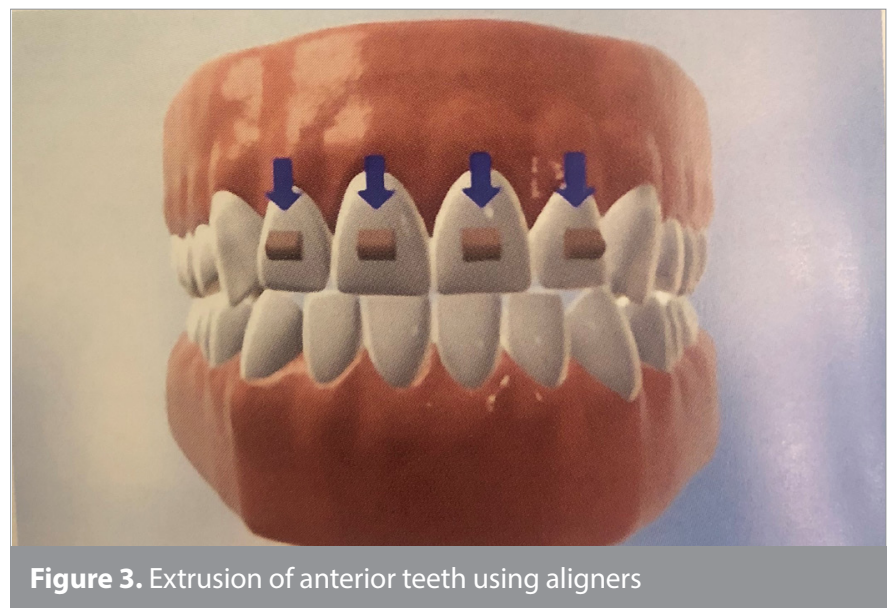


visalign group. The short duration of treatment with aligners was asserted with the absence of the finishing and detailing phase, which can take up to 6 months with fixed appliances. On the contrary, in extraction cases, Invisalign treatment duration is $44 \%$ longer as compared to fixed-appliance treatment (33). Patients with good compliance are required to visit the orthodontist in 10-12 week intervals in aligner therapy, whereas 4-6 week intervals are inevitable when treating with fixed appliances. Therefore, more appointments are required in fixed appliances therapies (38). Also, the chair time is found to be significantly shorter in clear aligners group, allowing the clinician to treat more patients $(38,39)$.

Effects of Clear Aligners on Periodontal Status and Oral Health As the number of adults treated with clear aligners increased, the periodontal effects of this treatment were found to be negative in the literature (40-42). Use of clear aligners facilitates oral hygiene, thus improving the periodontal status and causing a decrease in plaque levels, gingival inflammation, bleeding upon probing, and pocket depth $(2,40)$. Fixed appliances and wires made plaque control difficult and had adverse effects on periodontal tissues, making orthodontic treatment a predisposing factor for periodontal diseases (43). However, according to the study of Han et al. (40), with careful oral hygiene education and repeated plaque control, patients treated with fixed appliances and clear aligners showed similar gingival and plaque index. Clear aligners not only promote better oral hygiene, and better periodontal health but also reduce the plaque accumulation and the development of white spot lesions. According to the study of Azeem et al. (44), orthodontic treatment with clear aligners showed a low incidence of newly developed WSL's.

\section{Post Orthodontic Treatment Stability of Clear Aligners}

As in all types of orthodontic treatment, stability is one of the most important issues to discuss regarding clear aligners. One study investigated the post-retention stability outcomes of cases treated with clear aligners and fixed orthodontic appliances using the American Board of Orthodontics objective grading system (30). Retention protocol included only the use of removable thermoplastic Essix retainers and no fixed retainers were applied. Three years following the retention phase, relapse was seen in both groups in terms of total alignment, however, maxillary anterior leveling seemed to be stable in the fixed appliances group but relapsed in the Invisalign group (30). This data can only provide a preliminary insight for post-retention outcomes of clear aligners and the results cannot be generalized since only removable retention appliances were used and the researchers relied heavily on patient cooperation. Since clear aligner therapy is a relatively novel treatment method, retention studies regarding aligners are limited in the literature and further investigation is required on this subject $(16,30)$.

\section{Root Resorption and Clear Aligners}

Root resorption is one of the chief problems of orthodontic treatment and it is known that fixed orthodontic appliances can give rise to root resorption, generating excessive pressure at the apical level and causing external apical root resorption (45-48). However, few studies have assessed root resorption caused by thermoplastic aligners. A systematic review conducted in 2017 that could include only three studies concluded that aligners could also cause root resorption at the end of orthodontic treatment; however, the incidence and severity are lower as compared to fixed appliances (49). Another study stated that the incidence of root resorption caused by aligners is similar to the resorption caused by light orthodontic forces (50). According to the study by Gay et al. (49), $41.81 \%$ of teeth showed signs of apical root resorption after clear aligner treatment, with upper and lower incisors being the most affected teeth. This situation is explained by the root structure and the great extent of movement shown by the incisors.

\section{CONCLUSION}

- Clear aligners provide an esthetic and comfortable option to conventional fixed mechanics.

- Obtaining periodontal health is easier in patients treated with clear aligners and less white spot lesions develop during the treatment.

- Clear aligners can be used in mild to moderate crowding cases but caution must be exercised in complex cases.

- $\quad$ Root resorption is still a risk associated with orthodontic treatment in aligner therapy, such as in fixed appliances.

- $\quad$ Long term stability studies are required in this field.

Peer-review: Externally peer-reviewed.

Author Contributions: Concept - İ.T., E.Ö., G.M.; Design - İ.T., E.Ö., G.M.; Supervision - E.Ö., G.M.; Materials - I.T., E.Ö., G.M.; Data Collection and/ or Processing - I.T., E.Ö., G.M.; Analysis and/or Interpretation - I.T., E.Ö., G.M.; Literature Search - I.T., E.Ö., G.M.; Writing Manuscript - I.T., E.Ö., G.M.; Critical Review - I.T., E.Ö., G.M

Conflict of Interest: The authors have no conflict of interest to declare.

Financial Disclosure: The authors declared that this study has received no financial support.

\section{REFERENCES}

1. Weir T. Clear Aligners in Orthodontic Treatment. Aus Dent J 2017; 62: 58-62. [CrossRef]

2. Karkhanechi M, Chow D, Sipkin J, David S, Boylan R, Norman R, et al. Periodontal Status of adult patients treated with fixed buccal appliances and removable aligners over one year of active Orthodontic Theraphy. Angle Orthod 2013; 83: 146-51. [CrossRef]

3. Melkos AB. Advances in digital technology and orthodontics: a reference to the Invisalign method. Med Sci Monit 2005; 11: 39-42.

4. Malik $\mathrm{OH}$, McMullin A, Waring DT. Invisible orthodontics part 1: invisalign. Dent Update 2013; 40: 203-4, 207-10, 213-5. [CrossRef]

5. Graber TM. Orthodontics: Current Principles and Techniques. In: Paquette D, Colville C, Wheeler T, editors. Clear Aligner Treatment. St. Louis, Mosby, 2012, p.778-811.

6. Fujiyama K, Honjo T, Suzuki M, Matsuoka S, Deguchi T. Analysis of pain level in cases treated with Invisalign aligner: comparison with fixed edgewise appliance therapy. Prog Orthod 2014; 15: 64. [CrossRef]

7. Available from: hhtp:// www.invisalign.com/why-invisalign. Accessed October, 2013. 
8. Miller KB, McGorray SP, Womack R, Quintero JC, Perelmuter M, Gibson $J$, et al. A comparison of treatment impacts between Invisalign aligner and fixed appliance therapy during the first week of treatment. Am J Orthod Dentofacial Orthop 2007; 131: 302.e1-9. [CrossRef]

9. Proffit WR, Fields H, Sarver D. Contemporary Orthodontics. 5th ed. St. Louis: Mosby, 2013, p.355-7.

10. Drake C, McGorray S, Dolce C, Nair M, Wheeler T. Orthodontic Tooth Movement with Clear Aligners. ISRN Dent 2012; doi: 10.5402/2012/657973. [CrossRef]

11. Ponitz RJ. Invisible retainers. Am J Orthod 1971; 59: 266-72. [CrossRef]

12. Nahoum $\mathrm{H}$. Forces and moments generated by removable thermoplastic aligners. Am J Orthod Dentofacial Orthop 2014; 146: 545-6. [CrossRef]

13. Barone S, Paoli A, Razionale AV, Savignano R. Computational design and engineering of polymeric orthodontic aligners. Int J Numer Method Biomed Eng 2017; 33: doi: 10.1002/cnm.2839. [CrossRef]

14. Kim T, Öztürk Ortan Y. Clear Aligner Appliances: Fabrication and Clinical Application. Turk J Orthod 2009; 22: 256-66. [CrossRef]

15. Available from: www.ca-clear-aligner.com/en/b2c/index.html.

16. Gu J, Tang J, Skulsi B, Fields H, Beck F, Firestone A, et al. Evaluation of Invisalign treatment effectiveness and efficiency compared with conventional fixed appliances using the Peer Assessment Rating index. Am J Orthod Dentofacial Orthop 2017; 151: 259-66. [CrossRef]

17. Wong B. Invisalign A to Z. Am J Orthod Dentofacial Orthop 2002; 121: 540-1. [CrossRef]

18. Simon M, Keilig L, Schwarze J, Jung B, Bourauel C. Treatment outcome and efficacy of an aligner technique-regarding incisor torque, premolar derotation and molar distalisation. BMC Oral Health 2014; 14: 68. [CrossRef]

19. Lagravère MO, Flores-Mir $C$. The treatment effects of Invisalign orthodontic aligners: a systematic review. J Am Dent Assoc 2005; 136: 1724-9. [CrossRef]

20. Kravitz N, Kusnoto B, BeGole E, Obrez A, Agran B. How well does Invisalign work? A prospective clinical study evaluating the efficacy of tooth movement with Invisalign. Am J Orthod Dentofacial Orthop 2009; 135: 27-35. [CrossRef]

21. Boissere W, Morton J, Ojima K. Aligner Orthodontics: Diagnostics, Biomechanics Planning and Treatment. 2015 Hanover: Quintescence Publishing, p.26-30.

22. Sheridan JJ, McMinn R, LeDoux W. Essix thermosealed appliances: various orthodontic uses. J Clin Orthod 1995; 29: 108-13.

23. Ali SA, Miethke HR. Invisalign, an innovative invisible orthodontic appliance to correct malocclusions: advantages and limitations. Dent Update 2012; 39: 254-6, 258-60. [CrossRef]

24. Wheeler T. Orthodontic clear Aligner Treatment. Semin Orthod 2017; 23: 83-9. [CrossRef]

25. Hennessy J, Al-Awadhi EA. Clear aligners generations and orthodontic tooth movement. J Orthod 2016; 43: 68-76. [CrossRef]

26. Boyd RL. Periodontal and restorative considerations with clear aligner treatment to establish a more favorable restorative environment. Compend Contin Educ Dent 2009; 30: 280-2, 284, 286-8.

27. Chan E, Darendeliler M. The Invisalign appliance today: A thinking persons orthodontic appliance. Semin Orthod 2017; 23: 12-64. [CrossRef]

28. Turley PK. Evolution of esthetic considerations in orthodontics. Am J Orthod Dentofacial Orthop 2015; 148: 374-9. [CrossRef]

29. Hennesy J, Garvey T, Al-Awadhi E. A randomized clinical trial comparing mandibular incisor proclination produced by fixed labial appliances and clear aligners. Angle Orthod 2016; 86: 706-12. [CrossRef]

30. Kunchio D, Maganzini A, Shelton C, Freeman K. Invisalign and traditional orthodontic treatment post-retention outcomes compared using the American Board of Orthodontics objective grading system. Angle Orthod 2007; 77: 864-9. [CrossRef]

31. Djeu G, Shelton C, Maganzini A. Outcome assessment of invisalign and traditional orthodontic treatment compared with the American board of orthodontics objective grading system. Am J Orthod Dentofacial Orhop 2005; 128: 292-8. [CrossRef]

32. Kassas W, Al-Jewair T, Preston B, Tabba S. Assessment of Invisalign treatment outcomes using the $A B O$ model grading system. J World Fed Orthod 2013; 2: 61-4. [CrossRef]

33. Weihong L, Wang S, Zhang Y. The effectiveness of Invisalign Appliance in extraction cases using the $A B O$ model grading system: a multicenter randomized clinical trial. Int J Clin Exp Med 2015; 8: 8276-82.

34. Hönn M, Göz G. A Premolar Extraction case using the Invisalign System. J Orofac Orthop 2006; 67: 385-94. [CrossRef]

35. Rossini G, Parrini S, Castroflorio T, Deregibus A, Debernardi C. Efficacy of Clear Aligners in controlling Orthodontic Tooth Movement. Angle Orthod 2015; 85: 881-9. [CrossRef]

36. Yıldırım A. A prospective Study to determine the efficiency of Clear Aligner Appliance. Doctoral Dissertation, Istanbul University Institute of Health Sciences Orthodontics Department, 2013.

37. Skidmore KJ, Brook KJ, Thomson WM, Harding W. Factors influencing treatment time in orthodontic patients 2006 Am J Orthod Dentofacial Orhop 2006; 129: 230-8. [CrossRef]

38. Buschang PH, Shaw SG, Ross M, Crosby D, Campbell PM. Comparative time efficiency of aligner therapy and conventional edgewise braces. Angle Orthod 2014; 84: 391-6. [CrossRef]

39. Bradley TG. Changes in orthodontic treatment modalities in the past 20 years: exploring the link between technology and scientific evidence. J Ir Dent Assoc 2013; 59: 91-4.

40. Han JY. A comparative study of combined periodontal and orthodontic treatment with fixed appliances and clear aligners in patients with periodontitis. J Periodontal Implant Sci 2015; 45: 193204. [CrossRef]

41. Levrini L, Mangano A, Montanari P, Margherini S, Caprioglio A, Abbate GM. Periodontal health status in patients treated with the Invisalign $\left.{ }^{(}{ }^{\oplus}\right)$ system and fixed orthodontic appliances: A 3 months clinical and microbiological evaluation. Eur J Dent 2015; 9: 404-10. [CrossRef]

42. Abbate GM, Caria MP, Montanari P, Mannu C, Orrù G, Caprioglio A, et al. Periodontal health in teenagers treated with removable aligners and fixed orthodontic appliances. J Orofac Orthop 2015; 76: 240-50. [CrossRef]

43. Türkkahraman H, Sayin MO, Bozkurt FY, Yetkin Z, Kaya S, Onal S. Archwire ligation techniques, microbial colonization, and periodontal status in orthodontically treated patients. Angle Orthod 2005; 75: 231-6.

44. Azeem M, UI Hamid W. Incidence of White Spot lesions during Clear Aligner Theraphy. J World Fed Orthod 2017; 6: 127-30. [CrossRef]

45. Tieu LD, Saltaji H, Normando D, Flores-Mir C. Radiologically determined orthodontically induced external apical root resorption in incisors after non-surgical orthodontic treatment of class II division 1 malocclusion: a systematic review. Prog Orthod 2014; 15: 48. [CrossRef]

46. Iglesias-Linares A, Sonnenberg B, Solano B, Yañez-Vico RM, Solano E, Lindauer SJ, et al. Orthodontically induced external apical root resorption in patients treated with fixed appliances vs removable aligners. Angle Orthod 2017; 87: 3-10. [CrossRef]

47. Linge $L$, Linge $B O$. Patient characteristics and treatment variables associated with apical root resorption during orthodontic treatment. Am J Orthod Dentofacial Orthop 1991; 99: 35-43. [CrossRef]

48. Roscoe MG, Meira JB, Cattaneo PM. Association of orthodontic force system and root resorption: A systematic review. Am J Orthod Dentofacial Orthop 2015; 147: 610-26. [CrossRef] 
49. Gay G, Ravera S, Castroflorio T, Garino F, Rossini G, Parini S, et al. Root resorption during orthodontic treatment with Invisalign: a radiometric study. Prog Orthod 2017; 18: 12. [CrossRef]
50. Zheng M, Liu R, Ni Z, Yu Z. Efficiency, effectiveness and treatment stability of clear aligners: A systematic review and meta-analysis. Orthod Craniofac Res. 2017; 20: 127-133. [CrossRef] 